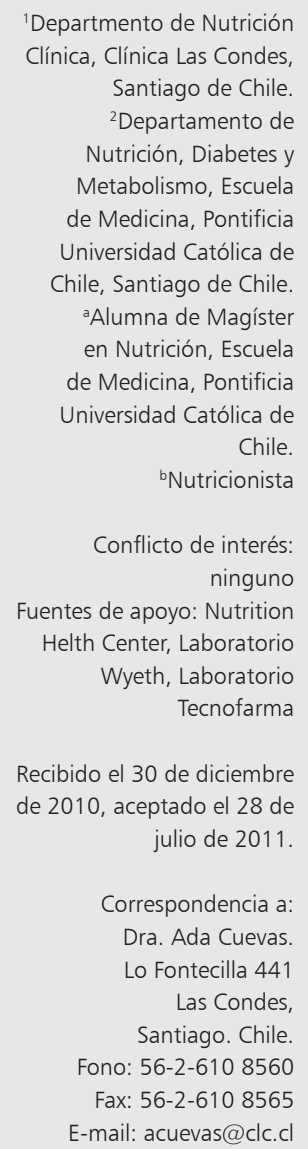

\section{Eficacia y seguridad de una dieta muy baja en calorías en un grupo de mujeres chilenas con sobrepeso u obesidad}

\author{
ADA CUEVAS ${ }^{1}$, MARÍA J. CORDERO ${ }^{2, a}$, CRISTINA OLIVOS ${ }^{1}$, \\ DANIELLA GHIARDO ${ }^{1, b}$, VERÓNICA ÁLVAREZ ${ }^{1}$
}

\section{Efficacy and safety of a very-low-calorie diet in a group of overweight or obese Chilean women}

Background: Very low calorie diets (VLCDs) have been used to induce a rapid decrease in total body weight. Aim: To evaluate the efficacy and safety of a VLCD in a group of overweight and obese Chilean women. Material and Methods: Thirty women with a body mass index $(B M I)>27 \mathrm{~kg} / \mathrm{m}^{2}$ were enrolled to a 4 weeks intervention with a VLCD (using liquid meal replacement). Anthropometric and metabolic parameters were evaluated at baseline and at the end of the intervention. Results: Twenty five women (83\%) completed the total intervention period. Average weight loss was $5.7 \pm 1.5 \mathrm{~kg}$ (-6.9\% of the initial weight) corresponding to a $34.7 \pm 13.4 \%$ of the excess of weight. Significant improvements in triglycerides, total cholesterol and LDL cholesterol (Low Density Lipoproteins) were observed. Insulin resistance determined by Homeostasis model assessment (HOMA), decreased significantly from $3.3 \pm 1.8$ to $2.0 \pm 0.9(p=0.003)$. No serious adverse events were reported. Conclusions: Short term use of VLCDs is safe and effective to induce rapid weight loss in Chilean women.

(Rev Med Chile 2011; 139: 1286-1291).

Key words: Caloric restriction; Diet; Obesity; Weight loss.

\section{L}

a obesidad es un problema de salud creciente e importante a nivel mundial, incluido $/$ Latinoamérica $^{1-3}$. Ha sido definida como una enfermedad crónica, caracterizada por un aumento de la grasa corporal, asociada a una alta morbilidad y mortalidad general ${ }^{1,4,5}$.

La reducción de peso está asociada a una mejoría significativa de las comorbilidades asociadas a la obesidad. Ha sido demostrado que incluso pequeñas reducciones, de 5 a $10 \%$ del peso corporal, inducen mejorías significativas en las anomalías metabólicas asociadas a la obesidad ${ }^{1,4-8}$. Sin embargo, la reducción de peso es difícil y desalentadora, debido a la alta tasa de fracasos y a las modestas reducciones de peso.

Las dietas de muy bajas calorías (VLCDs) han surgido como una adecuada opción para el tratamiento de la obesidad en pacientes seleccionados, especialmente, en aquellos que presentan alguna contraindicación para recibir tratamiento farmacológico o quirúrgico ${ }^{9-12}$. Estas dietas se definen como alimentos formulados con un contenido energético de entre 450 y 800 calorías diarias, los cuales constituyen la única fuente de energía y de todos los nutrientes esenciales necesarios que son requeridos en un programa de pérdida de peso. El objetivo de estas dietas es inducir una rápida baja de peso (1,5 a 2,5 kilogramos por semana), preservando la masa muscular, para lo cual se entrega un alto contenido de proteínas, de alto valor biológico; se debe aportar un mínimo de 50 gramos de proteínas y de 55 gramos de carbohidratos al día ${ }^{13}$.

Su mecanismo de acción es que al reducir la ingesta calórica diaria a menos de 800 calorías, se estimula el metabolismo lipídico como primera fuente energética y dado el alto aporte de proteínas de alto valor biológico se puede preservar la masa magra. Por otra parte, el aumento en la producción 
de cetonas, debido al bajo aporte de carbohidratos induce mayor saciedad, la que se manifiesta a pocos días de iniciada la dieta.

Una de las formas más utilizadas para lograr una dieta con estas características es el reemplazo de comidas con fórmulas proteicas líquidas. Estas últimas contienen proteínas de alta calidad biológica y parecen producir mayores bajas de peso, en comparación incluso a otras dietas muy bajas en calorías. Esto se ha atribuido a que los pacientes obesos subestiman su ingesta en aproximadamente 40 a 50\% (por la dificultad en estimar tamaño de las porciones, composición de macro-nutrientes, contenido calórico y recordatorio de comidas). En cambio estas fórmulas con una composición y cantidad constante y pre-determinada, minimizan este error.

Las VLCDs han sido usadas desde 1970 para inducir rápida baja de peso, sin embargo, al comienzo de su utilización se reportaron graves efectos adversos, incluso muertes asociados a su uso. Esto fue debido a que las proteínas de las primeras fórmulas eran de colágeno hidrolizado, el que carece de triptófano que es un aminoácido esencial y eran deficientes en vitaminas y minerales. Sin embargo, desde la introducción de fórmulas con proteínas de alto valor biológico (leche, huevo, soya), son consideradas seguras y efectivas si se usan en individuos obesos bien seleccionados y bajo supervisión médica, pudiendo utilizarse por períodos de hasta 12 a 16 semanas $^{9-13}$.

Los productos más utilizados en Europa y Estados Unidos de Norteamérica (EEUU) son Slimfast ${ }^{\circledR}$ y Optifast ${ }^{\circledR}$. Este último ha sido utilizado desde 1974 y cuenta con numerosos estudios que avalan su uso. En Chile, actualmente contamos con un producto de muy similar composición (Nutralean ${ }^{\circledR}$ ) que permite implementar este tipo de tratamientos.

Los meta-análisis de estudios a largo plazo concluyen que la baja de peso es mayor con este tipo de dieta si se compara con dietas hipocalóricas tradicionales. Varios estudios han concluido que la mayor baja de peso inicial con VLCD se asocia con una mayor baja de peso a largo plazo, asumiendo que los pacientes participen en un programa de mantención que incluye cambios de estilo de vida. Por otra parte, se ha evidenciado que inducen una baja de peso promedio de $22 \%, 12 \%$ en disminución de la glicemia, $12 \%$ disminución del colesterol total y $10 \%$ en reducción de la presión arterial.
Existe abundante evidencia acerca del uso de VLCDs tanto en Estados Unidos de Norteamérica ${ }^{9}$ como en Europa ${ }^{13}$, pero existe escasa evidencia acerca de su uso en población Latinoamericana y no existen estudios desarrollados previamente en Chile.

El objetivo de este estudio por tanto, es evaluar la eficacia y seguridad de esta dieta en un grupo de mujeres chilenas, con sobrepeso u obesidad.

\section{Materiales y Métodos}

\section{Sujetos}

Fueron reclutadas 30 mujeres chilenas con sobrepeso u obesidad (índice de masa corporal; IMC $>27 \mathrm{~kg} / \mathrm{m}^{2}$ ), entre 18 y 65 años, funcionarias de Clínica Las Condes. Los criterios de exclusión incluyeron participantes con enfermedades concomitantes (diabetes mellitus tipo 1, infecciones severas, enfermedades malignas, falla renal o hepática, enfermedad cardiovascular, porfiria, desórdenes psiquiátricos o neurológicos, abuso de alcohol u otras sustancias), mujeres embarazadas o en lactancia, presencia de algunas alteraciones metabólicas (niveles de glucosa plasmática $>110$ $\mathrm{mg} / \mathrm{dL}$, triglicéridos $>250 \mathrm{mg} / \mathrm{dL}$ ), ser considerado por el investigador como no apto para el estudio, haber participado previamente en otro programa, tratamiento o serie clínica controlada en los últimos 90 días antes de ingresar al estudio.

El estudio fue revisado y aprobado por la Comisión de Investigación de la Dirección Académica de la Clínica Las Condes, luego de considerar que los procedimientos respetaban las normas éticas de la Declaración de Helsinki.

\section{Diseño del estudio}

Todas las participantes fueron evaluadas semanalmente por personal entrenado. El peso, la talla y la circunferencia de cintura (CC) fueron medidos y se calculó el IMC. La presión arterial fue medida en el brazo derecho, con un manguito adecuado y por la misma persona, usando un esfigmomanómetro de mercurio estándar. $\mathrm{Al}$ inicio y al final del estudio, se tomaron muestras de sangre venosa, luego de 12 horas de ayuno, para mediciones bioquímicas (pruebas hepáticas, electrolitos, glicemia, insulinemia, uricemia, nitrógeno ureico y lípidos) usando los procedimientos estándar.

También se les realizó un electrocardiograma 
de reposo, a todas las pacientes, al inicio y al término de la intervención.

Para el plan de alimentación de muy bajas calorías se utilizó un preparado comercial de reemplazo (Nutralean ${ }^{\circledR}$, ver composición nutricional en anexo 1) durante cuatro semanas. El menú diario consistía en 3 a 4 batidos proteicos ( 3 batidos para aquellas pacientes con IMC entre 27 y $35 \mathrm{~kg} / \mathrm{m}^{2}$ y 4 batidos a aquellas con IMC $\geq 35 \mathrm{~kg} /$ $\mathrm{m}^{2}$ ), además de 2 porciones de vegetales, con $5 \mathrm{ml}$ de aceite, distribuidos al almuerzo y la cena. Se les permitió consumir una cantidad libre de líquidos como agua, sopas, jugos y gelatinas sin azúcar. Adicionalmente, se prescribió suplementación con vitaminas y minerales (Centrum ${ }^{\circledR} 1$ comprimido al día). Con esto se aportó alrededor de 600 a 750 calorías diarias con 65 a 80 g de proteínas de alto valor biológico (equivalente a un aporte proteico aproximado a 0,7 a $1,0 \mathrm{~g} / \mathrm{kg}$ de peso corporal).

No se indicó ningún tipo especial de ejercicio físico, sino sólo que continuaran con su actividad física habitual.

Cada participante fue controlada semanalmente para evaluar la adherencia a la dieta, signos vitales, peso, CC y eventos adversos. Las mediciones fueron realizadas por una misma persona y con los mismos equipos calibrados. Para chequear la adherencia dietaria, en cada control se realizó una medición cualitativa de cetonuria.

\section{Análisis estadístico}

En este estudio, el peso, el IMC, la CC, la presión arterial (PA), el perfil lipídico, la glicemia de ayuno y la resistencia insulínica (RI) fueron medidas basalmente y luego de las 4 semanas de intervención. Se calcularon los cambios en relación a los niveles basales.

Se utilizó t-test pareado para comparar los cambios de las variables medidas basalmente y luego de la intervención. El test de los signos de Wilcoxon se utilizó cuando la distribución era distinta a la normal. Todos los análisis fueron realizados usando el software SigmaStat 3.0. Todos los test se realizaron de dos colas y considerando 0.05 como nivel de significancia.

\section{Resultados}

\section{Sujetos}

Un total de 30 mujeres fueron incluidas en el estudio (IMC $32,6 \pm 3,7 \mathrm{~kg} / \mathrm{m}^{2}$ ), pero 5 de ellas se retiraron luego de la primera semana de intervención (tres de ellas porque les disgustó el batido, una porque presentó intolerancia digestiva (náuseas y distensión abdominal) y otra por motivos personales no relacionados con el estudio). Las 25 restantes completaron las cuatro semanas y asistieron a todos sus controles. En la Tabla 1 se describen las características y mediciones antropométricas basales de las mujeres seleccionadas.

\section{Eficacia}

Los porcentajes de reducción, desde el nivel basal, en peso corporal, IMC, CC, PAS (presión arterial sistólica) y PAD (presión arterial diastólica)

Tabla 1. Características basales de las mujeres seleccionadas

\begin{tabular}{|llr|}
\hline & \multicolumn{3}{c|}{ Media \pm DE (Mín - Máx) } \\
\hline Edad (años) & $38 \pm 9,7$ & $(19-56)$ \\
\hline Peso $(\mathrm{kg})$ & $82,9 \pm 11,4$ & $(66,3-116,5)$ \\
\hline Talla $(\mathrm{m})$ & $1,59 \pm 0,06$ & $(1,46-1,72)$ \\
IMC $\left(\mathrm{kg} / \mathrm{m}^{2}\right)$ & $32,6 \pm 3,7$ & $(27,7-43,5)$ \\
\hline CC $(\mathrm{cm})$ & $101,9 \pm 9,1$ & $(84-120)$ \\
\hline
\end{tabular}

DE, Desviación Estándar; Mín, Mínimo; Máx, Máximo.

Tabla 2. Cambios antropométricos

\begin{tabular}{|lcccc|}
\hline & Basal (Media \pm DE ) & Final (Media \pm DE ) & \% Cambio (\%) & Valor $\mathbf{p}$ \\
\hline Peso $(\mathrm{kg})$ & $82,7 \pm 10,5$ & $77,0 \pm 9,8$ & $-6,89$ & $<0,001$ \\
IMC $\left(\mathrm{kg} / \mathrm{m}^{2}\right)$ & $32,2 \pm 3,3$ & $30,0 \pm 3,0$ & $-6,83$ & $<0,001$ \\
CC $(\mathrm{cm})$ & $101,9 \pm 8,8$ & $92,0 \pm 7,4$ & $-9,72$ & $<0,001$ \\
PAS $(\mathrm{mmHg})$ & $120,6 \pm 10,0$ & $110,7 \pm 12,8$ & $-8,2$ & $<0,001$ \\
\hline PAD $(\mathrm{mmHg})$ & $77,8 \pm 6,8$ & $72,1 \pm 8,0$ & $-7,33$ & $<0,002$ \\
\hline
\end{tabular}


fueron todos estadísticamente significativos. Los resultados se muestran en la Tabla 2. Los cambios del nivel basal y final de peso corporal, CC e IMC, se muestran en la Figura 1.

\section{Parámetros metabólicos}

Los cambios en los parámetros metabólicos más importantes, luego de la intervención, se muestran en la Tabla 3. Hubo mejorías significativas en el perfil lipídico, insulinemia y RI (determinada por el índice de HOMA). Al inicio del estudio 5 y 8 pacientes presentaban niveles séricos elevados de fosfatasas alcalinas y gamma glutamil transferasa (GGT), respectivamente; estos se normalizaron completamente al terminar la intervención, observándose una disminución significativa en los niveles de estas dos enzimas plasmáticas.

\section{Seguridad}

Se reportaron algunos efectos adversos menores que tendieron a disminuir a lo largo de la intervención (entre paréntesis se indica el número de pacientes que los presentó): constipación (9), náuseas (8), distensión abdominal (7), fatiga (6), cambios anímicos (5), diarrea (4), calambres (3), vómitos (1), mareos (1), cefalea (1), piel seca (1), cabello opaco (1), boca amarga (1).

No se reportaron efectos adversos severos. No se observaron cambios significativos en los niveles plasmáticos de electrolitos, transaminasas, ni ácido úrico. No se registraron cambios clínicamente relevantes de signos vitales, examen físico o electrocardiograma durante el estudio.

\section{Adherencia}

La adherencia evaluada de acuerdo a le medición de cetonuria fue de $71 \%$ después de la primera semana de intervención y de $62 \%$ al completar el estudio (60\% y 77\% después de la segunda y tercera semana respectivamente). No hubo diferencias significativas en la baja de peso

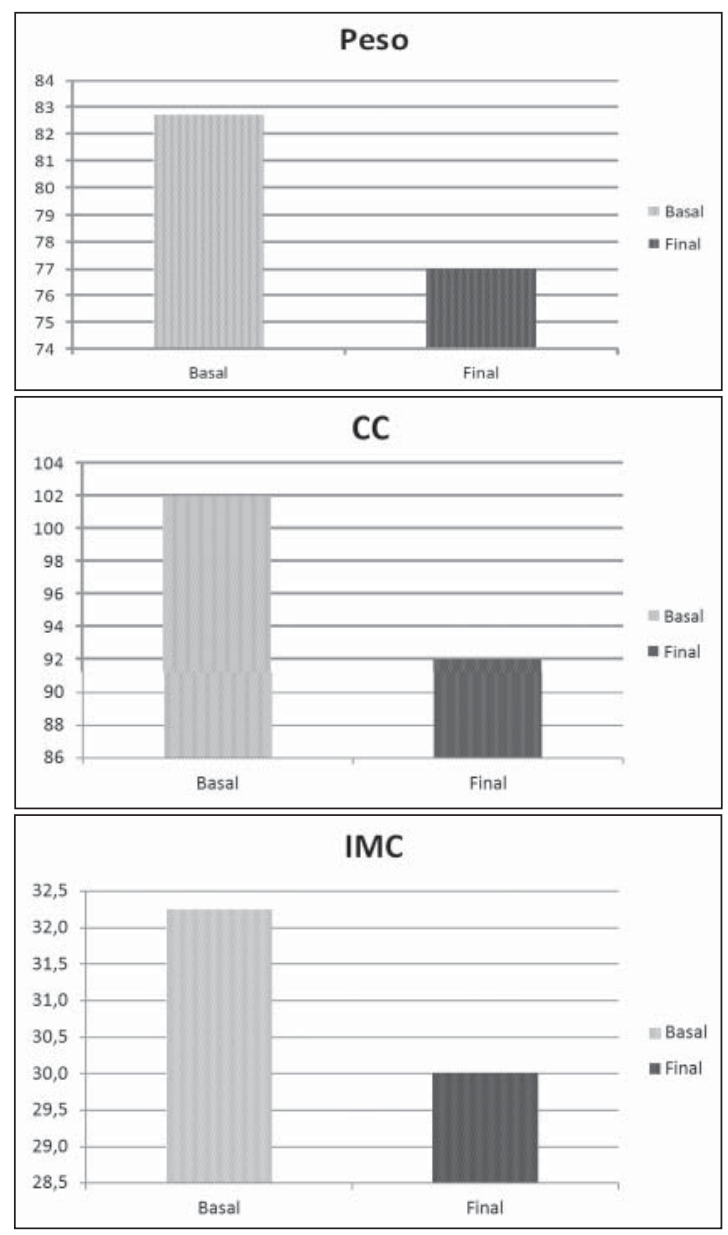

Figura 1. Cambio Peso corporal, CC e IMC desde nivel basal.

Tabla 3. Cambios en parámetros metabólicos

\begin{tabular}{|lcccc|}
\hline & Basal & Final & \% Cambio & Valor p \\
\hline C-Total $(\mathrm{mg} / \mathrm{dl})$ & $187,6 \pm 31,3$ & $149,1 \pm 25,7$ & $-20,52$ & $<0,001$ \\
\hline C-LDL $(\mathrm{mg} / \mathrm{dl})$ & $114,8 \pm 27,0$ & $90,1 \pm 19,0$ & $-21,52$ & $<0,001$ \\
\hline C-HDL $(\mathrm{mg} / \mathrm{dl})$ & $48,7 \pm 11,5$ & $42,1 \pm 10,7$ & $-13,55$ & $<0,001$ \\
\hline Triglicéridos $(\mathrm{mg} / \mathrm{dl})$ & $120,7 \pm 52,4$ & $84,4 \pm 29,0$ & $-30,07$ & 0,005 \\
\hline Glicemia $(\mathrm{mg} / \mathrm{dl})$ & $95,4 \pm 14,4$ & $92,8 \pm 1,8$ & $-2,73$ & 0,548 \\
Insulinemia $(\mathrm{uU} / \mathrm{ml})$ & $13,7 \pm 6,9$ & $8,7 \pm 3,2$ & $-36,50$ & 0,002 \\
\hline HOMA & $3,3 \pm 1,8$ & $2,0 \pm 0,9$ & $-39,39$ & 0,003 \\
\hline
\end{tabular}


(medida en kilogramos) entre aquellas con y sin cetonuria, medida después de la cuarta semana $(5,9 \pm 1,2$ vs $5,1 \pm 2,3 ; \mathrm{p}=0,14)$.

\section{Discusión}

Actualmente, la obesidad es un importante problema de salud pública y en constante aumento en los países latinoamericanos, incluido Chile, donde $27 \%$ y $39 \%$ de la población presenta obesidad y sobrepeso respectivamente ${ }^{15}$.

Lamentablemente, el tratamiento de la obesidad es difícil y poco alentador, debido a la baja adherencia a las dietas tradicionales, a la alta frecuencia de reganancia del peso perdido y a la escasez de fármacos disponibles para el tratamiento de la obesidad. De ahí que surja la necesidad de implementar nuevas estrategias terapéuticas para optimizar los resultados en baja de peso y corrección de morbilidades asociadas.

En nuestro estudio hemos evidenciado que las VLCDs son efectivas y seguras en mujeres chilenas con obesidad o sobrepeso. Siguiendo esta dieta en que consumían bebidas proteicas en reemplazo de las principales comidas, las pacientes lograron una significativa reducción de su peso corporal, equivalente a una pérdida de $7 \%$ del peso corporal.

Adicionalmente, se observó una reducción significativa del perímetro abdominal asociada a una significativa mejoría en distintos parámetros metabólicos, incluyendo los niveles de triglicéridos, colesterol total y colesterol LDL. También se obtuvo una reducción significativa de la resistencia insulínica. Se observó una caída del colesterol HDL, lo cual es frecuente de ver en dietas hipocalóricas que restringen el consumo de grasa, incluidas las VLCDs. Esta reducción, en general, es transitoria y se produce durante el período de intervención, luego tiende a aumentar, ${ }^{9,10,14}$.

Estos resultados son comparables a intervenciones a corto plazo realizadas en otras poblaciones, principalmente de EEUU y Europa, donde se han reportado pérdidas de peso que van desde 0,7 a 2,5 kg por semana, dependiendo del aporte calórico y del IMC inicial, y que están asociadas a mejorías significativas de parámetros metabólicos, incluidos: PA, lípidos plasmáticos, glicemia, RI y mejoría o resolución de esteatosis hepática, entre otros $^{9-13}$. Estos mismos estudios han evidenciado la seguridad de estas dietas cuando son indicadas y supervisadas adecuadamente.
En nuestro estudio, la dieta fue bien tolerada durante el período de intervención y no se reportaron efectos adversos mayores, sin embargo, y de acuerdo a la medición de cetonurias, se observa una tendencia a una menor adherencia al término de la dieta en comparación a la evaluación después de la primera semana. Esto podría atribuirse principalmente a la monotonía de la indicación dietaria. De ahí entonces que la utilidad de las VLCDs radica principalmente en una estrategia inicial en un programa de reducción de peso, dado que tienen la ventaja de que al inducir una importante baja de peso inicial generan una gran motivación en el paciente. Por otra parte, al ser pobres en carbohidratos generan cetosis lo cual reduce el apetito y mejora la adherencia. También el uso de reemplazos de comidas, con porciones y composiciones predeterminadas tiene mejores resultados en baja de peso y adherencia en comparación a otras intervenciones, dado que frecuentemente los pacientes obesos tienden a subestimar su ingesta calórica ${ }^{19}$.

Además, se ha evidenciado que con un adecuado tratamiento de mantención multidisciplinario, la mantención de baja de peso lograda con la VLCDs es equivalente o incluso superior a la obtenida con otras dietas de bajas calorías.

No obstante es importante mencionar que estas dietas tienen limitaciones en su uso, y solamente son seguras si son indicadas y supervisadas por profesionales con conocimiento de los aspectos fisiológicos y psicológicos que ocasiona una rápida baja de peso. Deben ser indicadas en pacientes seleccionados, estando contraindicado su uso en pacientes portadores de enfermedades renales y hepáticas, arritmias cardiacas, diabetes tipo 1, enfermedades psiquiátricas o trastornos de la conducta alimentaria, lactancia, embarazo, niños $\mathrm{y}$ ancianos.

Además, sólo pueden ser utilizadas por un tiempo limitado (hasta 12 a 16 semanas), dado que la adherencia disminuye con el tiempo y por los posibles efectos adversos asociados a su uso prolongado.

Nuestro estudio tiene algunas limitaciones; todas las participantes eran funcionarias de la Clínica Las Condes, lo cual puede interpretarse como un sesgo de selección dado que podríamos especular que estas personas tuvieron una mejor adherencia a la intervención y a sus controles. Segundo, el período de intervención fue corto (sólo 
de 4 semanas), lo cual limita la interpretación de nuestros resultados sólo a ese período y no al largo plazo, y finalmente este estudio no evaluó la reganancia de peso después de haber finalizado la intervención, por lo que no es posible especular lo que sucederá con estas pacientes a largo plazo.

En conclusión, este estudio ha evidenciado que las VLCDs son efectivas y seguras en un período corto de intervención en un grupo de mujeres chilenas con obesidad o sobrepeso.

Estudios adicionales son necesarios en otras poblaciones latinoamericanas, así como también estudios de intervención más prolongados que evalúen la mantención de la baja de peso lograda.

\section{Referencias}

1. OMS. Obesity: Preventing and managing the global epidemic. Report of a WHO consultation. Ginebra, OMS, 1998.

2. James WP. The epidemiology of obesity: the size of the problem. J Intern Med 2008; 263(4): 336-52. Review.

3. Delpeuch F, Maire B. Obesity and developing countries of the south. Med Trop (Mars) 1997; 57(4): 380-8. Review.

4. Clinical Guidelines on the Identification, Evaluation, and Treatment of Overweight and Obesity in Adults - The Evidence Report. Obes Res 1998; 6 Suppl 2: 51S-209S. Review.

5. Tsigos C, Hainer V, Basdevant A, Finer N, Fried M, Mathus-Vliegen $\mathrm{E}$ et al. Management of obesity in adults: European clinical practice guidelines. Obes Facts 2008; 1 (2): 106-16.

6. Case CC, Jones PH, Nelson K, O'Brian Smith E, Ballantyne CM. Impact of weight loss on the metabolic syndrome. Diabetes Obes Metab 2002; 4(6): 407-14.

7. Rössner S. Defining success in obesity management. Int J Obes Relat Metab Disord 1997; 21 Suppl 1: S2-4. Review.

8. Goldstein DJ. Beneficial health effects of modest weight loss. Int J Obes Relat Metab Disord 1992; 16(6): 397-415. Review.

9. National Task Force on the Prevention and Treatment of Obesity. Very low-calorie diets. JAMA 1993; 270 (8): 967-74. Review.

10. Mustajoki P, Pekkarinen T. Very low energy diets in the treatment of obesity. Obes Rev 2001; 2(1): 61-72. Review.

11. Delbridge E, Proietto J. State of the science: VLED (Very Low Energy Diet) for obesity. Asia Pac J Clin Nutr 2006; 15 (Suppl): 49-54. Review.

12. Tsai AG, Wadden TA. The evolution of very-low-calorie diets: an update and meta-analysis. Obesity 2006; 14(8): 1283-93. Review.
13. SCOOP-VLCD Working Group. Collection of data on products intended for use in very-low-calorie-diets. Disponible en: http://www.foodedsoc.org/scoop.pdf. [Consultado el 19 de noviembre de 2010].

14. Pekkarinen T, Takala I, Mustajoki P. Weight loss with very-low-calorie diet and cardiovascular risk factors in moderately obese women: one-year follow-up study including ambulatory blood pressure monitoring. Int J Obes Relat Metab Disord 1998; 22 (7): 661-6.

15. Ministerio de Salud/Instituto Nacional de Estadísticas. Encuesta Nacional de Salud 2009-2010. Disponible en: http://www.encuestasalud.cl/pdf/InformeENS_2009-2010_CAP5.pdf. [Consultado el 10 de mayo de 2011].

16. Vio F, Albala C. Nutrition transition in Chile: a case study. In: Globalization of food systems in developing countries: impact on food security and nutrition. FAO Food and Nutrition Paper 2004; 83: 275-84.

17. Albala C, Vio F, Kain J, Uauy R. Nutrition transition in Latin America: The case of Chile. Nutrition Reviews: 2001; 59 (6): 170-6.

18. Vio F. Prevención de la obesidad en Chile. Rev Chil Nutr: 2005; 32 (2): 80-7. Disponible en: http://www.scielo.cl/ scielo.php?pid=S0717-75182005000200001\&script=sci_ arttext. [Consultado el 19 de noviembre de 2010].

19. Lichtman SW, Pisarska K, Berman ER, Pestone M, Dowling $\mathrm{H}$, Offenbacher E et al. Discrepancy between self-reported and actual caloric intake and exercise in obese subjects. N Engl J Med 1992; 327(27): 1893-8.

Anexo 1. Composición Nutralean ${ }^{\circledR}$

\begin{tabular}{|l|l|l|}
\hline $\begin{array}{l}\text { Porción: } \mathbf{2} \text { medidas } \\
\text { colmadas (40 g) }\end{array}$ & $\mathbf{1 0 0} \mathbf{~ g}$ & $\mathbf{1}$ Porción \\
\hline Energía (Kcal) & 379 & 152 \\
Proteínas (g) & 36,7 & 14,7 \\
Grasa total (g) & 5,5 & 2,2 \\
Grasa Saturada (g) & 2,5 & 1,0 \\
Grasas Monoinsaturada (g) & 2,1 & 0,84 \\
Grasa Poliinsaturada (g) & 1,3 & 0,52 \\
Ácidos grasos trans (g) & 0,14 & 0,06 \\
Colesterol (mg) & 100 & 40 \\
H. de carbono disp. (g) & 41,1 & 16,4 \\
Azúcares (g) & 3,0 & 1,2 \\
Lactosa Fibra dietética total (g) & 2,35 & 0,9 \\
$\quad$ Fibra soluble (g) & 7,3 & 2,9 \\
Inulina (g) & 5,0 & 2,9 \\
Sodio (mg) & 360 & 144 \\
Potasio (mg) & 1.118 & 447 \\
\hline
\end{tabular}

